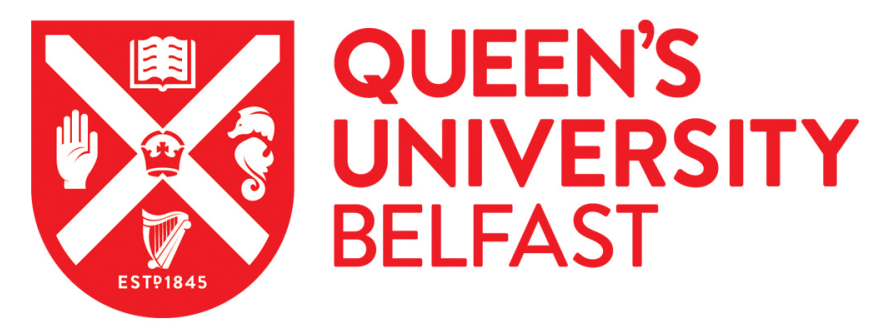

\title{
Investigating Approaches to Three-Dimensional Printing of Hydroxyapatite Scaffolds for Bone Regeneration
}

Zhou, Z., Buchanan, F., Lennon, A., \& Dunne, N. (2015). Investigating Approaches to Three-Dimensional Printing of Hydroxyapatite Scaffolds for Bone Regeneration. Key Engineering Materials, 631, 306-311 . https://doi.org/10.4028/www.scientific.net/KEM.631.306

\section{Published in:}

Key Engineering Materials

Document Version:

Peer reviewed version

Queen's University Belfast - Research Portal:

Link to publication record in Queen's University Belfast Research Portal

Publisher rights

Copyright 2014 Trans Tech Publications Ltd.

\section{General rights}

Copyright for the publications made accessible via the Queen's University Belfast Research Portal is retained by the author(s) and / or other copyright owners and it is a condition of accessing these publications that users recognise and abide by the legal requirements associated with these rights.

Take down policy

The Research Portal is Queen's institutional repository that provides access to Queen's research output. Every effort has been made to ensure that content in the Research Portal does not infringe any person's rights, or applicable UK laws. If you discover content in the Research Portal that you believe breaches copyright or violates any law, please contact openaccess@qub.ac.uk. 


\title{
Investigating Approaches for Three-Dimensional Printing of Hydroxyapatite Scaffolds for Bone Regeneration
}

\author{
Zuoxin Zhou ${ }^{1, a}$, Fraser Buchanan ${ }^{1, b}$, Alex Lennon ${ }^{1, c}$ and Nicholas Dunne ${ }^{1, d, ~ * ~}$ \\ ${ }^{1}$ School of Mechanical and Aerospace Engineering, Queen's University Belfast, BT9 5AH, UK \\ az.zhou02@qub.ac.uk, bf.buchanan@qub.ac.uk, ca.lennon@qub.ac.uk, dn.dunne@qub.ac.uk
}

Keywords: Hydroxyapatite, Polyvinyl alcohol, Maltodextrin, 3D printing, Additive manufacturing, Architectural accuracy, Micro-CT, Image registration

\begin{abstract}
This study investigated the feasibility of manufacturing hydroxyapatite (HA)-based scaffolds using 3D printing technology by incorporating different binding additives, such as maltodextrin and polyvinyl alcohol (PVOH), into the powder formulation. Different grades of PVOH were evaluated in terms of their impact on the printing quality. Results showed that scaffolds with high architectural accuracy in terms of the design and excellent green compressive strength were obtained when the PVOH (high viscosity) was used as the binding additive for HA.
\end{abstract}

\section{Introduction}

Additive Manufacturing (AM) has been increasingly recognised as one of the most promising technology for the future production of tissue-engineering scaffolds. Among the available AM techniques, powder-based ink-jet $3 \mathrm{D}$ printing (3DP) has been employed to manufacture hydroxyapatite (HA) bone scaffolds, through a setting reaction between HA powder and acidic binder. However, delivering such binder in the 3DP system will result in premature failure of the printhead. To ensure long-term sustainability of the 3DP process, water-based binder solution would be preferable.

However due to the low reactivity between HA and water-based binder an efficient binding additive is required, which should have the ability to react with water-based binder and subsequently provide sufficient binding strength to HA to form rigid scaffolds.

Possible binding additives include maltodextrin and polyvinyl alcohol (PVOH), as suggested by some other studies ${ }^{[1,2]}$. Maltodextrin is a partially hydrolysed starch that is usually produced with a dextrose equivalent value lower than 20, whereas PVOH is synthesised via incomplete hydrolysis of poly(vinyl acetate), resulting in a small amount of acetate groups in the polymer chain network. Due to the nature of these two materials, both of them are easily dissolved in deposited water and can potentially achieve high printing strength after water evaporates.

In this study, the use of maltodextrin and $\mathrm{PVOH}$ as the binding additive for the development of HA-based powder formulation for 3DP manufacturing has been investigated. The effects of different additives and mixing ratios on the key printing properties, such as green compressive strength and architectural accuracy compared to the original CAD design were evaluated.

\section{Materials and Methods}

\section{Materials}

Hydroxyapatite powder (Captal ${ }^{\circledR}$, Plasma Biotal Ltd., UK) having a PSD of $\mathrm{D}_{10}=45.68 \mu \mathrm{m}$, $\mathrm{D}_{50}=67.84 \mu \mathrm{m}$, and $\mathrm{D}_{90}=94.40 \mu \mathrm{m}$ was used in this study as the bulk material ${ }^{[3]}$. It was blended with one of the following in-bed binding additives: (1) maltodextrin powder (419680, Sigma-Aldrich, UK) or (2) PVOH powder (either GM-14S or GH-20S, Nippon Gohsei, Japan).

The two PVOH powders differed in degree of polymerisation, which was indicated by viscosity. According to the manufacturer's data-sheet, GM-14S had viscosity of 23.0 29.0MPa.s and GH-20S had that of 40.0 46.0MPa.s, meaning GM-14S had a relatively lower degree of polymerisation than GH-20S. 
Prior to blending, the additive powders were milled and sieved in order to reduce the particle size to levels appropriate for the 3DP process. Maltodextrin was milled using a planetary mill (Pulverisette 6, Fritsch, Achern, Germany) with a milling cycle of $2 \mathrm{~min}$ on, $5 \mathrm{~min}$ rest and repeating 5 times. The rotational speed was set to 300rpm. After milling, the powders were sieved to pass through a $90 \mu \mathrm{m}$ mesh but retained by a $32 \mu \mathrm{m}$ mesh. Consequently the powder was within the particle size range of 32 90 $\mathrm{m}$, which allowed the setup of layer thickness $=100 \mu \mathrm{m}$ and also avoided excessive agglomeration.

A more robust milling procedure was required for PVOH. The rotational speed was increased to 600rpm and a milling cycle was set to $5 \mathrm{~min}$ on, $5 \mathrm{~min}$ rest and repeating 5 times. After milling, the obtained PVOH powder was also sieved to pass through a $90 \mu \mathrm{m}$ mesh and retained by a $32 \mu \mathrm{m}$ mesh.

Each of the in-bed binding additives was blended with the HA powder at ratios of 10:90 wt.\%, 20:80 wt.\%, and 30:70 wt.\%, with HA being the principal component. As such, nine different powders were prepared for the following 3DP manufacturing. Powder blending was conducted using a High Speed Mixer (Rondol, UK) with the cycle of $2 \mathrm{~min}$ on, $2 \mathrm{~min}$ rest, and repeating 3 times. The rotational speed was set to 1600rpm.

\section{DP}

A rectangular porous structure (pore size $=1.2 \mathrm{~mm} \times 1.2 \mathrm{~mm}$ ) and a cylinder block structure (Ø6mm x 12mm) (Fig. 1) were designed using Solidworks (Dassault Systèmes SolidWorks Corp, USA) and then manufactured for each HA:additive powder combination using 3DP. Water-based liquid (ZB7, Z Corporation, UK) was used as the binder material. After printing, the samples were dried at approximately $70^{\circ} \mathrm{C}$ for $1 \mathrm{~h}$ and then de-powdered.

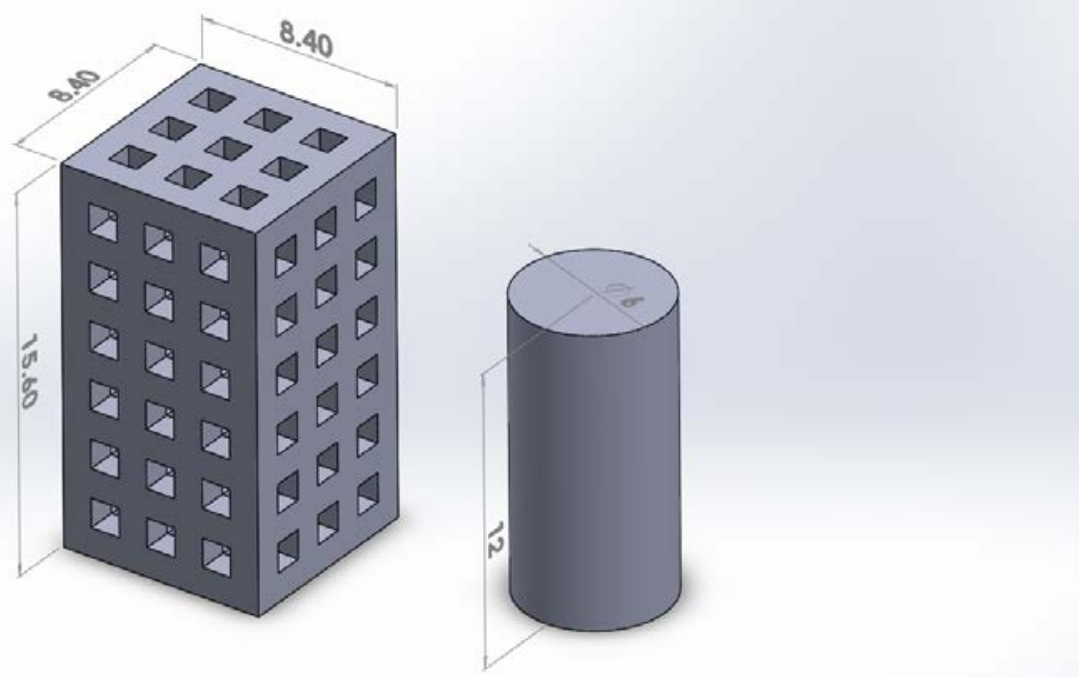

Fig. 1: CAD models of the structures that were designed for 3DP manufacturing

\section{Green Compressive Strength}

Compression tests were conducted on solid blocks using a universal materials test system (EZ50, Lloyds Instruments, UK) using a $5 \mathrm{kN}$ load cell at a rate of displacement of $0.5 \mathrm{~mm} / \mathrm{min}$. Each test was completed when the load reduced to $80 \%$ of the peak load. The peak load was used to calculate the green compressive strength for each specimen.

\section{Architectural Accuracy}

Scaffold geometries were scanned using a SkyScan 1174 compact desktop $\mu$-CT scanner system (SkyScan N.V., Belgium). The scanning protocol was as followings: pixel size $=16.25 \mu \mathrm{m}$, rotational step $=0.5^{\circ}$, exposure time at each step $=5.5 \mathrm{~s}$, and rotation $=360^{\circ}$. Reconstruction of the transmission images was performed using SkyScan NRecon software (Version 1.6.3.1), which generated a dataset of cross-sectional images. In order to quantify the level of architectural accuracy, the cross-sectional images from the manufactured object was compared to the reference image from the 3D CAD design. 
Image registration was needed to ensure alignment of the different images into one coordinate system.

The reference image was drawn with the same dimensions to the corresponding cross-section of the CAD design. The target images were obtained from the $\mu$-CT dataset following reconstruction. Five cross-sections for each scaffold were used as the target images for analysing the architectural accuracy. Their locations were selected to ensure a uniform distribution within the scaffold geometry.

Both the reference image and the target image were imported in ImageJ software (National Institutes of Health, USA). Initially, the target image required thresholding as it was in 8-bit format. The reference image did not require thresholding due to its black and white format. Scale set was performed post thresholding. The $\mu$-CT image had a pixel size of $16.25 \mu \mathrm{m}$, which was used to set the scales. For the reference image, a line was drawn on the bottom boundary and the scale was then set by defining its length as $8,400 \mu \mathrm{m}$.

Thereafter, these two images were registered using rigid registration. The target image was selected to transform and the reference image was used as the template. The algorithm was run under the following setup: $n$ initial positions to attempt $=1$; tolerance $=1.000$; level $=4$; and stop-level $=2$. On completion of registration, a difference image was generated with non-overlapping areas shown in white (grayscale $=255$ ) and overlapping areas shown in black (grayscale $=0$ ) $($ Fig. 2). The number of pixels that carried either grayscale value was quantified from the histogram of the difference image. Printing accuracy was then calculated by the ratio of the number of black pixels to the number of all pixels (Eq. 1).

$$
\text { Printing Accuracy }(\%)=\text { black pixels } /(\text { black pixels }+ \text { white pixels }) \times 100 \%
$$

Where: black pixels and white pixels = the number of black and white in the difference image, respectively.

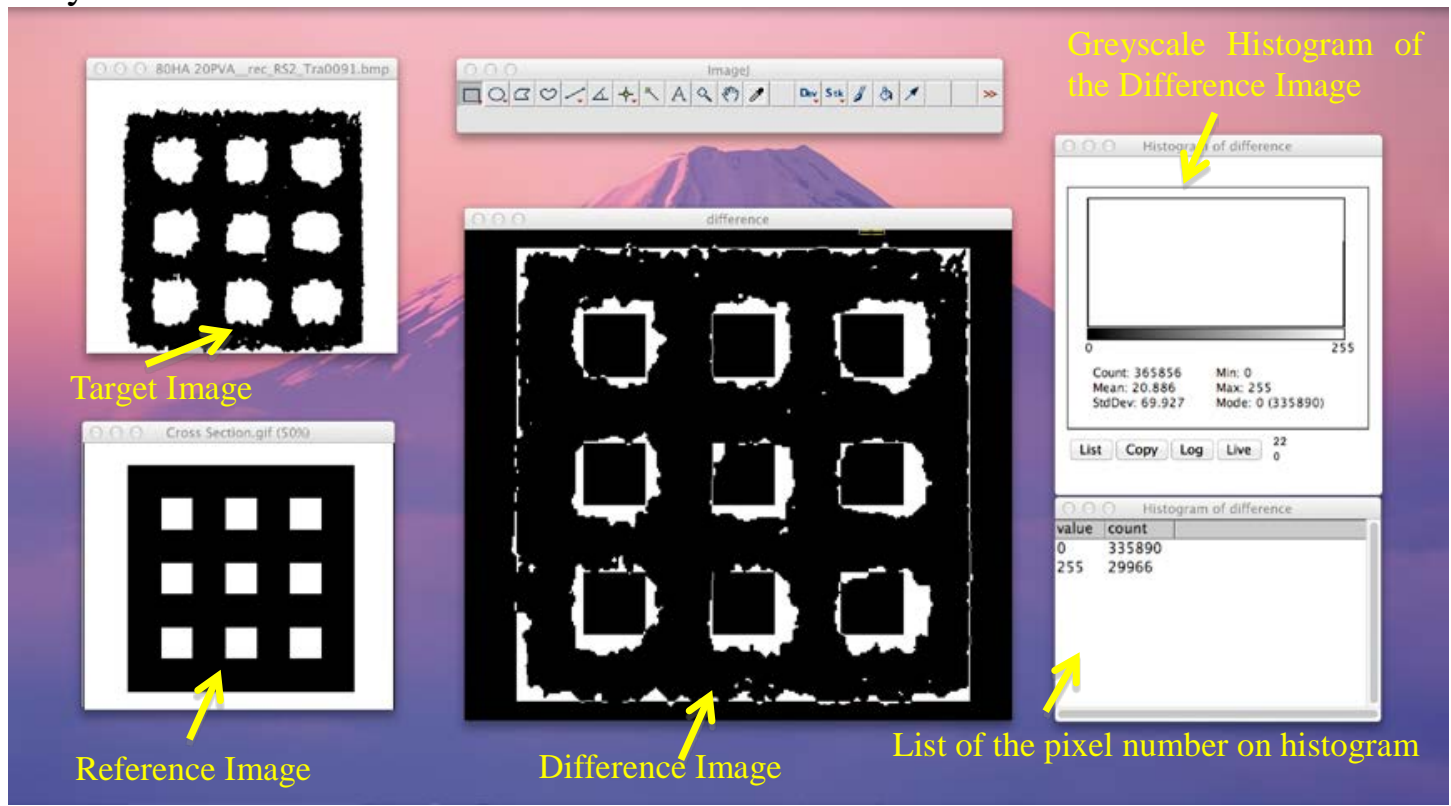

Fig. 2: Operational interface of image registration (ImageJ software). A difference image from a $\mu-C T$ scanning image and the reference image was showed and its greyscale histogram was generated.

\section{Statistical Analysis}

Data collected from all the experimental tests was evaluated for statistical significance using a one-way Analysis of Variance (ANOVA) followed by a post-hoc Tukey's HSD test for the comparison between each group. A value of $\mathrm{p}<0.05$ was considered to be significantly different. Data that was approximately normal distributed was decided on basis of normal probability tests. Tests 
were conducted using Minitab 14 student software (Minitab, Inc., USA) and SPSS 13.0 software (SPSS, USA).

\section{Results and Discussion}

\section{DP Manufacturing}

The unbounded powders within the structure of HA:maltodextrin scaffolds adhered strongly to each other. Unbound powders could not be removed from the structure using only compressed air. Therefore a fine needle used to loosen powders before they could be removed using compressed air (Fig. 3). This problem occurred for all the three HA:maltodextrin powders processed, irrespective of the HA:maltodextrin ratio. However, using a needle to improve the patency of the 3DP scaffolds should be avoided as it may cause irreparable damage to the surrounding structure. Consequently, the 70:30wt.\% HA:maltodextrin scaffolds were characterised in order to make comparisons to the HA:PVOH scaffolds.
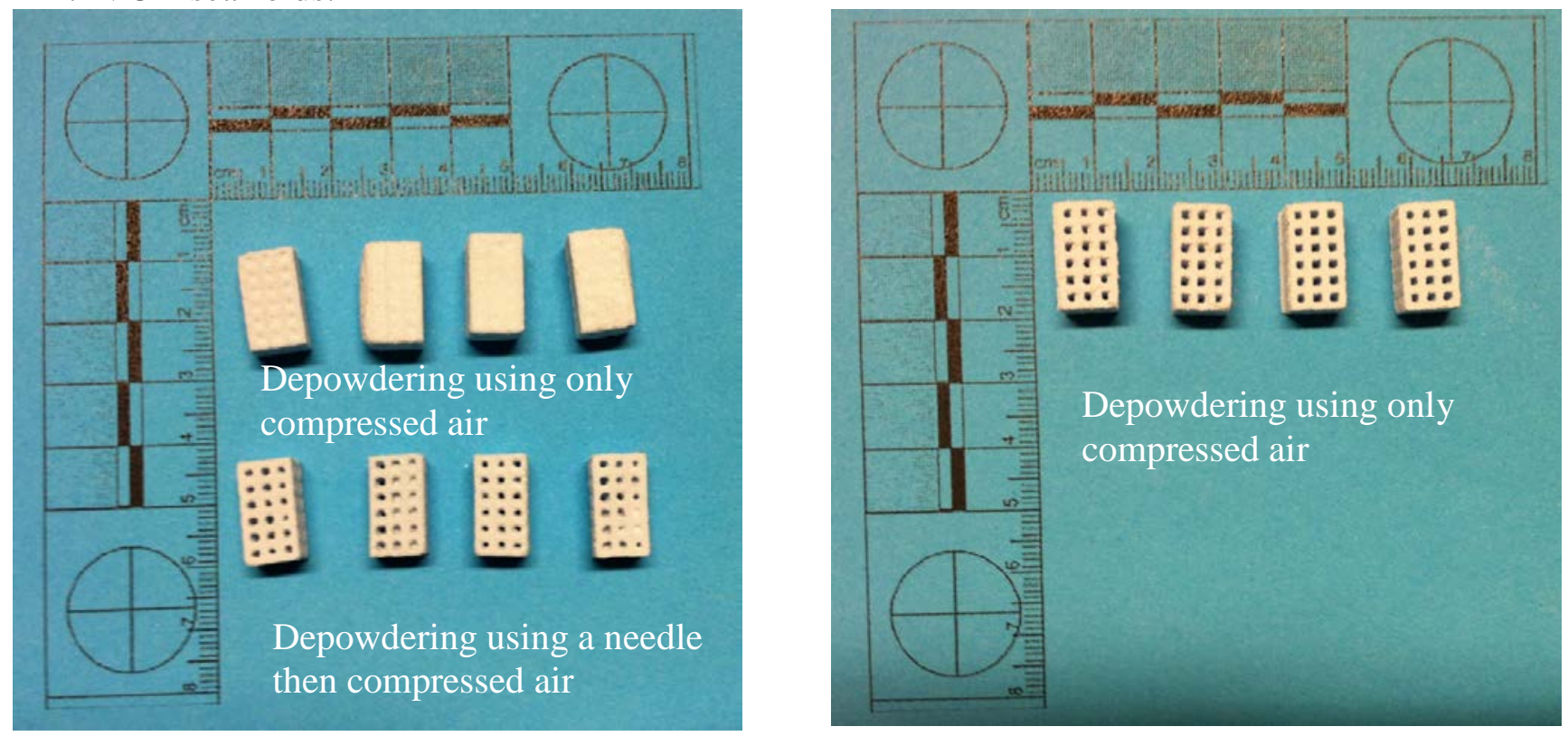

Fig. 3: (left) 70:30wt.\% HA:maltodextrin scaffolds, indicating the difficulties in depowdering these scaffolds post 3DP process; and (right) 70:30wt.\% HA:GH-20S samples

This depowdering problem was not evident during manufacture of the HA:PVOH scaffolds. The unbounded powders could be easily using compressed air, without significant damage of the scaffold architecture. The scaffolds had sufficient green strength for handling processes, with the exception of the scaffolds manufactured from the 90:10wt.\% HA: GM-14S powder.

Consequently, scaffolds manufactured from the following three powder formulations were not included in characterisation: 90:10wt.\% HA:maltodextrin, 80:20wt.\% HA:maltodextrin, and 90:10wt.\% HA:GM-14S.

\section{Green Strength}

Considering the 3DP scaffolds tested, those manufactured from the 70:30wt.\% HA:GH-20S powder combination had the greatest green compressive strength $(5.63 \pm 0.27 \mathrm{MPa})$ (Fig. 4$)$. These 3DP scaffolds demonstrated a green compressive strength that was significantly higher $(\mathrm{p}<0.05)$ than the for the HA:maltodextrin samples $(3.70 \pm 0.47 \mathrm{MPa})$ and HA:GM-14S $(0.37 \pm 0.01 \mathrm{MPa})$ scaffolds manufactured at the same powder ratio. As the ratio of GH-20S decreased, the green compressive strength of HA:GH-20S samples decreased significantly $(\mathrm{p}<0.05)$. The HA:GM-14S samples exhibited the lowest green compressive strength when considered in the context of all the scaffolds tested. 


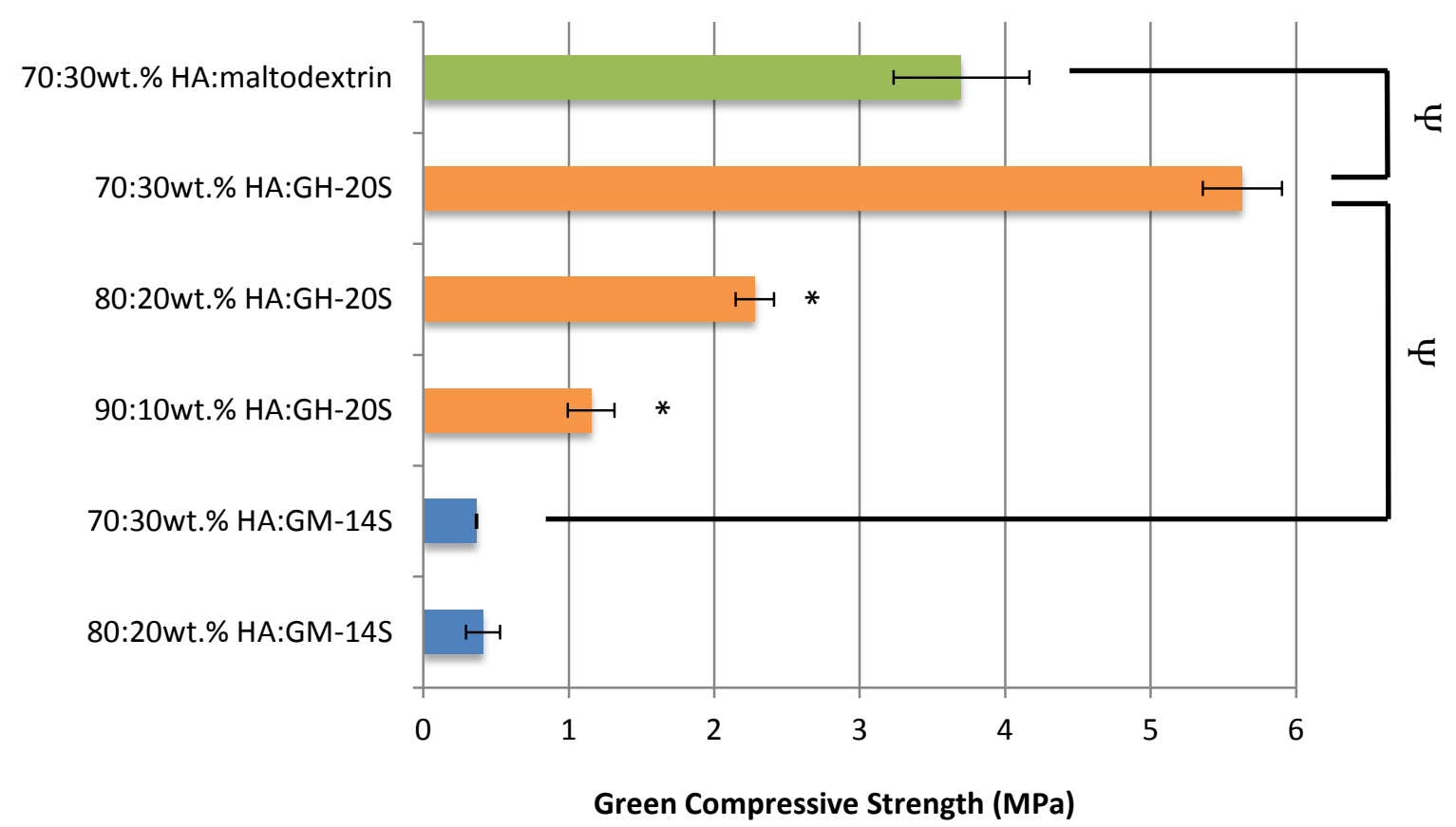

Fig. 4: Green compressive strength (mean \pm SD) of the solid scaffolds manufactured using the different powder formulations. * denotes p-values $<0.05$, indicating a significant difference between powders having differing HA:additive ratios; $\Psi$ denotes p-values $<0.05$, indicating a significant difference between powders consisting differing binding additives

The degree of polymerisation plays an important role in determining the characteristics of PVOH. An increase in the degree of polymerisation can potentially result in a higher viscosity and greater tensile strength ${ }^{[4]}$. These properties are particularly favorable for 3DP applications as PVOH can act as a binding additive for the HA powder particles. PVOH with higher solution viscosity could offer a higher level of stability during the 3DP process. Post dehydration, the PVOH membrane demonstrating a higher strength facilitates a stronger level of binding to the surrounding HA powder particles.

\section{Architectural Accuracy}

The HA:maltodextrin scaffolds exhibited two problems with respect to its geometry: (1) the overall shape did not conform to the initial design; and (2) pores were of random shapes and sizes. In contrast, both HA:GH-20S and HA:GM-14S scaffolds showed better architectural accuracy compared to the HA:maltodextrin scaffolds at the same mixing ratio (70:30wt.\%) (Table 1). Their overall shapes were clearly square, which matched the initial 3D CAD design. The shape and size of the pores were highly consistent for $\mathrm{HA}: \mathrm{PVOH}$ scaffolds. Therefore, at the same mixing ratio (70:30wt.\%), the 3DP scaffolds manufactured using maltodextrin as the binding additive showed significantly lower architectural accuracy $(\mathrm{p}<0.05)$ than its PVOH counterparts. The HA:maltodextrin scaffold exhibited a architectural accuracy of $78.82 \pm 1.43 \%$, which suggested approximately $22 \%$ mismatch between its structure and the initial 3D CAD design.

The major problem in using the maltodextrin as a binding additive was the excessive wetting, which caused difficulties in depowdering and poor architectural accuracy. The excessive wetting of maltodextrin affected powders in the unbounded area. The unbounded powders were partially wetted by the liquid, which made it difficult to remove following the 3DP process. Even though pores within the structure could be forced to open using some means (e.g. fine needle), they were of random shapes and sizes, which were poorly matched the initial 3D CAD design. The $\mu-C T$ scanning image supported the excessive wetting of the maltodextrin as the majority of the struts in the structure were larger than the initially defined size of $1.2 \mathrm{~mm}$. This wetting behaviour significantly deteriorated the architectural accuracy of the 3DP scaffolds, which restricted the control and prediction of their performance. 
Table 1: Architectural accuracy of HA-based scaffolds using differing binding additives (30wt.\%)

\begin{tabular}{|c|c|c|c|}
\hline Material & Cross-Sectional Image & Difference Image & $\begin{array}{c}\text { Architectural Accuracy } \\
\text { Mean } \pm \text { SD }\end{array}$ \\
\hline HA:Maltodextrin & & & $78.82 \pm 1.19 \%$ \\
\hline HA:GH-20S & & & $85.68 \pm 1.43 \%$ \\
\hline HA:GM-14S & & & $84.60 \pm 0.68 \%$ \\
\hline
\end{tabular}

\section{Conclusion}

Using maltodextrin as the binding additive in the 3DP manufacture of scaffolds caused an issue with respect to depowdering, resulting in poor architectural accuracy compared to the initial 3D design. High architectural accuracy was achieved for the porous scaffolds manufactured from HA:PVOH powder formulations.

The effects of degree of polymerisation had a strong influence on the green compressive strength of 3DP scaffolds. PVOH having a higher degree of polymerisation (GH-20S) demonstrated a higher binding strength to HA, which resulted in higher green compressive strength.

Overall, 3DP scaffolds demonstrating a high architectural accuracy and excellent green compressive strength were obtained using GH-20S as binding additive for HA. This study has demonstrated an alternative approach that has the potential for the production of high-quality HA scaffolds using 3DP technology.

\section{References}

[1] R. Chumnanklang, T. Panyathanmaporn, K. Sitthiseripratip, J. Suwanprateeb, 3D printing of hydroxyapatite: Effect of binder concentration in pre-coated particle on part strength, Mat Sci Eng C. 27 (2007) 914-921.

[2] Y. shanjani, D. Croos, J. Amritha, R.M. Pilliar, R.A. Kandel, E. Toyserkani, Solid freeform fabrication and characterization of porous calcium polyphosphate structures for tissue engineering purposes, J Biomed Mater Res. 93 (2010) 510-519.

[3] Z. Zhou, F. Buchanan, C. Mitchell, N. Dunne, Printability of calcium phosphate: calcium sulfate powders for the application of tissue engineered bone scaffolds using the 3D printing technique, Mat Sci Eng C. 38 (2014) 1-10.

[4] G. Yahya, S. Ali, M. Al-Naafa, E. Hamad, Preparation and viscosity behavior of hydrophobically modified poly (vinyl alcohol) (PVA), J Appl Polym Sci. 57 (1995) 343-352. 